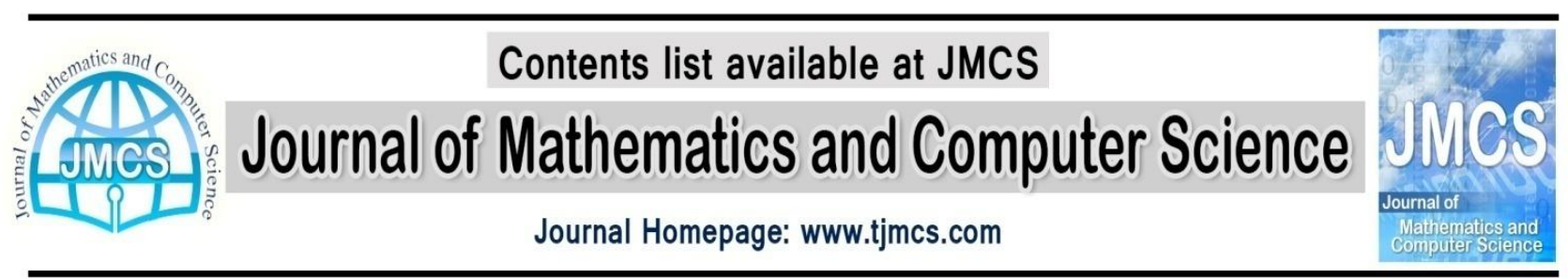

\title{
Conditional p-maxian problem on network
}

\author{
Somayeh Ziyanloo , Jafar Fathali \\ Department of Mathematics, Shahrood University, Iran \\ ziyanloo@yahoo.com,fathali@shahroodut.ac.ir
}

Article history:

Received July 2013

Accepted August 2013

Available online August 2013

\begin{abstract}
This article investigates the problem of conditional p-maxian on the networks. Assume a network like $G=(V, E)$ that the weight in each of its vertices $v_{i}$ is positive $w_{i}$. In a $p$ maxian problem, the goal is to find a set contains $\mathrm{p}$ as the vertice of the set and as a facility; in a way that the sum of maximum of weighted distance of other point is the highest one from the set.

In the problem of conditional p-maxian, we assume that some facilities are already existed and there is need to add other $\mathrm{p}$ ones.

In this article, other new algorithms are presented to solve the problem of conditional p-maxian and their results (e.g. their solution time in finding the optimum points) are compared. One of the applications of this article is to determine an optimum place for landfill wastes. The wastes must be in the farthest distance of the demand (areas that people live) and it must be in the appropriate places to serve the requests.
\end{abstract}

Keywords: p-maxian, Conditional, Location, Network. 


\section{Introduction}

The time when the problems are solved, is considered very important. Inevitably, the less the time is, the more optimum can be obtained in terms of time and place. The purpose in this paper is to determine optimum location for a few facilities using a solution with less time and it is assumed that there are a few facilities available previously in the network.

A network such as $G=(V, E)$ is given such that each vertice $V_{i}$ has $W_{i}$ weight. In the pmedian targeted problem , finding a set including network vertice $\mathrm{p}$ as an agent to provide service, is proposed such that the least value is obtained for the total weighted intervals of other points from this set. Berman et al [1,2] have solved the conditional p-median problem and they have assumed that some more service provider $\mathrm{p}$ must be added to the previously available ones to give the service on the network.

Burkard et al have explored the p-maxian problem [3]. The purpose in this problem is to find a set including service provider $\mathrm{p}$ as the vertice of the network such that maximum total weighted interval is achieved from other points to this point. Some service providers are present previously in the network in the conditional p-maxian problem and the purpose is to find a set including network vertice $\mathrm{p}$ as service provider such that maximum value is obtained for the total maximum weighted interval from other points to the service providers.

In this paper, we have extended the Berman-Derzner [1] as well as Berman-Simchi [2] algorithms for the conditional p-maxian problem and these algorithms are compared to find optimum points on the network in order to locate optimum place for the facilities on the network with the lowest time. The lowest time solution (to find optimum location for facilities) leads to the cost effective ways in terms of time and expenses, so it is considered important and in practice, it extends the optimization concept, particularly and considerably when there are many demanding points and a lot of facilities.

\section{The required initial concepts definitions}

A network such as $G=(V, E)$ is assumed in which each vertice $V_{i}$ has positive weight $W_{i}$. A set of demanding points $\mathrm{V}$ as vertices is assumed with $|\mathrm{V}|=\mathrm{n}$ and $\mathrm{E}$ is considered as a set of edges. The shortest interval between any $x, y \in G$ is $d_{x y} \cdot|Q|=q$ includes the previously available service providers in the network. In the conditional p-maxian problem, the purpose is to maximize following function:

$\mathrm{F}(\mathrm{x})=\sum w_{i} \max \left\{d\left(v_{i}, x_{j}\right)_{1 \leq j \leq p}, d\left(v_{i}, y_{j}\right)_{1 \leq j \leq q}\right\}$

Where $x_{j}$ defines the location of new service provider $j$ and $j=1, \ldots \ldots, p$ and $y_{j}$ denotes the place of available service provider $\mathrm{j}$ and $\mathrm{j}=1, \ldots \ldots, \mathrm{q}$.

For solving the conditional $\mathrm{p}$-maxian problem with two algorithms, it is assumed that $\mathrm{p}$ service providers are located on the vertices of the network and the previously available service providers 
are not required to be located on the vertices of the network and these can be located on every points of the network.

The available service provider is defined as the previously available service provider and the new service provider is defined as the one we planned to find its optimum location in the network.

\section{The problem definition}

In the conditional p-maxian problem, the purpose is to find a place for the new facility $\mathrm{p}$ on the vertices of the network where there are some previously available facilities on the network.

Therefore, we plan to find a place for a new service provider on the network such that on total, maximum weighted interval is obtained for other points from service providers. In this paper, we solve the conditional p-maxian problem by changing it into unconditional mode.

\section{The proposed solution}

In this paper, we determine optimum place for new facilities on vertices of the network using interval matrix and it is shown in the appropriate algorithm. The conditional p-maxian problem has been changed into unconditional $(\mathrm{p}+1)$-maxian problem in the first algorithm and it has been changed into unconditional p-maxian problem in the second algorithm and these algorithms are compared (in terms of solution time). Now, solution algorithms for conditional p-maxian problem are explained and an example is given.

\subsection{The first algorithm}

Berman and Simchi have proposed the first algorithm [2] for solving the conditional p-median problem and we have extended it for the conditional p-maxian problem. The interval matrix in this algorithm for solving conditional p-maxian problem includes rows similar to demanding points (vertices) as well as columns similar to the vertices on which potential points can be located for new service providers (potential locations are those vertices from the network that lacks any pre available facility). In this algorithm, the interval matrix D is given by assuming that the new column $\mathrm{a}_{0}$ is a pre available service provider $\mathrm{q}$.

Matrix derivatives are measured as $d_{i j}$ with the shortest distance from the demanding point $i$ (vertice) to the new service provider location $\mathrm{j}$ (potential location $\mathrm{j}$ ). For column $\mathrm{a}_{0}$ the derivatives are measured as $\max \left\{\mathrm{d}_{\mathrm{ik}}\right\}_{\mathrm{k} \in \mathrm{Q}}$ where $\mathrm{Q}$ is the previously available service providers and $\mathrm{d}_{\mathrm{ik}}$ denotes the lowest distance from the demanding point $\mathrm{i}$ (vertice) to the location of the available service provider $\mathrm{k}$.

Matrix of $D$ for the conditional p-maxian equals to $n \times(n+1)$ when $y_{i} \notin N$ with $i=1, \ldots q(N$ denotes vertices of network and $y_{i}$ denotes available location for facility $\left.i\right)$ and matrix of $D$ for the conditional $p$-maxian equals to $n \times(n-q+1)$ when $y_{i} \in N$ with $i=1, \ldots . q$.

In this algorithm, conditional p-maxian is changed into unconditional (p+1)-maxian problem. 


\subsection{The second algorithm}

The second algorithm was proposed by Berman and Dersner and we have extended it for conditional p-maxian problem. In this algorithm, we have modified distance D for conditional pmaxian problem solution. Thus, matrix rows are counterparts to demanding points (vertices) and matrix columns are counterparts to the vertices where new service providers can be placed on potentially. In this mode, the modified distance matrix is shown as $\widetilde{\mathrm{D}}$ and its derivatives are measured as follows:

$\widetilde{\mathrm{D}}_{\mathrm{ij}}=\max \left\{\tilde{\mathrm{d}}_{\mathrm{ij}}, \max \left\{\tilde{\mathrm{d}}_{\mathrm{ik}}\right\}_{\mathrm{k} \in \mathrm{Q}}\right\}$

$\widetilde{D}$ matrix for conditional $p$-maxian problem equals to $n \times n$ when $y_{i} \notin N$ with $i=1, \ldots \ldots q$ and

$\widetilde{D}$ matrix for conditional $p$-maxian problem equals to $n \times(n-q)$ when $y_{i} \in N$ with $i=1, \ldots . . q$.

In this algorithm, conditional p-maxian problem is solved as a unconditional p-maxian one.

\subsection{Example}

Consider following network:

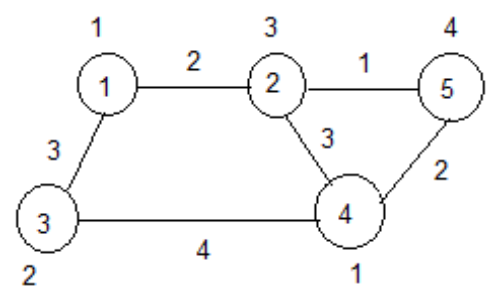

Figure 1. A network with five demanding points

There are 5 demanding points on a node with vertice weights and distances between two vertices are written on them.

Assume that $\mathrm{Q}=\{2,3\}$ (the present facilities are available on the nodes 2 and 3). The goal is to solve conditional 1-maxian problem?

The targeted function rate in conditional 1-maxian problem using following formula:

$\mathrm{F}(\mathrm{x})=\sum_{\mathrm{i}=1}^{5} \mathrm{w}_{\mathrm{i}} \max \left\{\mathrm{d}\left(\mathrm{v}_{\mathrm{i}}, \mathrm{x}_{\mathrm{j}}\right)_{1 \leq \mathrm{j} \leq 1}, \mathrm{~d}\left(\mathrm{v}_{\mathrm{i}}, \mathrm{y}_{\mathrm{j}}\right)_{1 \leq \mathrm{j} \leq 2}\right\}$

Is as follows: when new facility is located on node 1 :

$\mathrm{F}(1)=1 \times 3+3 \times 5+2 \times 5+1 \times 5+1 \times 5+4 \times 6=57$

When new facility is located on node 4 :

$\mathrm{F}(4)=1 \times 5+3 \times 5+2 \times 5+1 \times 4+4 \times 6=58$

Finally, when the new facility is located on node 5:

$\mathrm{F}(5)=1 \times 3+3 \times 5+2 \times 6+1 \times 4+4 \times 6=58$

Therefore, optimum locations for new facility are node 4 or node 5 based on above formula. In result, the facilities are located on nodes $4,3,2$ or nodes $5,3,2$. 
Solution for conditional 1-maxian problem by the first method:

Table 1: D distance matrix

\begin{tabular}{|c|lccc|}
\hline Demanding points & \multicolumn{4}{|l|}{ Potential locations } \\
\cline { 2 - 5 } & 1 & 4 & 5 & $\mathrm{a}_{0}$ \\
\hline 1 & 0 & 5 & 3 & 3 \\
2 & 2 & 3 & 1 & 5 \\
3 & 3 & 4 & 6 & 5 \\
4 & 5 & 0 & 2 & 4 \\
5 & 3 & 2 & 0 & 6 \\
\hline
\end{tabular}

In this method, the conditional 1-maxian problem is changed into unconditional 2-maxian and it is solved as follows:

Table 2: Distance matrix

\begin{tabular}{|l|lcccc|}
\hline $\begin{array}{l}\text { Demanding } \\
\text { points }\end{array}$ & \multicolumn{4}{l}{ Potential locations } \\
\cline { 2 - 6 } & $1, \mathrm{a}_{0}$ & $2, \mathrm{a}_{0}$ & $3, \mathrm{a}_{0}$ & $4, \mathrm{a}_{0}$ & $5, \mathrm{a}_{0}$ \\
\hline 1 & 3 & 3 & 3 & 5 & 3 \\
2 & 5 & 5 & 5 & 5 & 5 \\
3 & 5 & 5 & 5 & 5 & 6 \\
4 & 5 & 4 & 4 & 4 & 4 \\
5 & 6 & 6 & 6 & 6 & 6 \\
\hline
\end{tabular}

The matrix elements in table 2 are calculated as below:

$\mathrm{D}_{\mathrm{ij}}=\max \left\{\mathrm{d}_{\mathrm{ij}}, \max \left\{\mathrm{d}_{\mathrm{ik}}\right\}_{\mathrm{k} \in \mathrm{Q}}\right\}$

$\mathrm{a}_{0}$ denotes the location of pre available service provider. Therefore, $\mathrm{a}_{0}=\{2,3\}$.

Calculation of the targeted function using the table 2 when the problem is changed into unconditional 2-maxian form:

When the facilities are located on $1, \mathrm{a}_{0}$ :

$1 \times 3+3 \times 5+2 \times 5+1 \times 5+1 \times 5+4 \times 6=57$

When the facilities are located on $2, \mathrm{a}_{0}$ :

$1 \times 3+3 \times 5+2 \times 5+1 \times 4+4 \times 6=56$

When the facilities are located on $3, \mathrm{a}_{0}$ :

$1 \times 3+3 \times 5+2 \times 5+1 \times 4+4 \times 6=56$

When the facilities are located on $4, \mathrm{a}_{0}$ :

$1 \times 5+3 \times 5+2 \times 5+1 \times 4+4 \times 6=58$

When the facilities are located on $5, \mathrm{a}_{0}$ :

$1 \times 3+3 \times 5+2 \times 6+1 \times 4+4 \times 6=58$

So, in the unconditional maxian problem 2 , the optimum locations are on node $4, \mathrm{a}_{0}$ or node $5, \mathrm{a}_{0}$. In the other words, the facilities are located on nodes $3,2,4$ or $3,2,5$. 
The conditional 1-maxian problem solution by the second method:

Table 3. Distance $\widetilde{\mathrm{D}}$ matrix

\begin{tabular}{|c|lll|}
\hline Demanding points & \multicolumn{3}{|l|}{ Potential locations } \\
\cline { 2 - 4 } & 1 & 4 & 5 \\
\hline 1 & 3 & 5 & 3 \\
2 & 5 & 5 & 5 \\
3 & 5 & 5 & 6 \\
4 & 5 & 4 & 4 \\
5 & 6 & 6 & 6 \\
\hline
\end{tabular}

The elements of matrix in table 3 are measured as follows:

$\widetilde{\mathrm{D}}_{\mathrm{ij}}=\max \left\{\mathrm{d}_{\mathrm{ij}}, \max \left\{\mathrm{d}_{\mathrm{ik}}\right\}_{\mathrm{k} \in \mathrm{Q}}\right\}$

Conditional 1-maxian problem is changed into unconditional 1-maxian problem by above method.

Calculations of targeted function by $\widetilde{\mathrm{D}}$ distance matrix are as below:

When new facility is located on node 1 :

$1 \times 3+3 \times 5+2 \times 5+1 \times 5+1 \times 5+4 \times 6=57$

When new facility is located on node 4 :

$1 \times 5+3 \times 5+2 \times 5+1 \times 4+4 \times 6=58$

When new facility is located on node 5 :

$1 \times 3+3 \times 5+2 \times 6+1 \times 4+4 \times 6=58$

So, optimum location for new facility is located on node 4 or node 5 , as well. In result, the optimum locations for facilities are located on nodes $4,3,2$ or nodes 5,3,2.

\subsection{Result}

Now, the two algorithms are compared. In the first algorithm, the solution for conditional pmaxian problem was changed into the unconditional $(\mathrm{p}+1)$-maxian problem. But in the second algorithm, the solution for conditional $\mathrm{p}$-maxian problem was changed into the unconditional $\mathrm{p}$ maxian problem.

The solutions for conditional p-maxian problem using above algorithms for finding facilities optimum locations are the same.

In the first algorithm, the numbers of calculations are higher and therefore, it takes more time to find optimum locations for the facilities.

So, it is more efficient to use the second algorithm in order to find optimum locations for new facilities. And we need less time to find optimum locations for new facilities on vertices of the network. In result, the optimization has been adopted in time.

unconditional p-maxian problem on tree can be solved in linear time as it has been conducted in [3]. 


\section{References}

1. O. Berman, Z. Drezner, A new formulation for the conditional p-median and p-center problems, Operations Research Letters 36 (2008) 481-483.

2. O. Berman, D. Simchi-Levi, The conditional location problem on networks, Transportation Science 24 (1994) 77-78.

3. R. E. Burkard, J. Fathali, H. Taghizadehkakhki, The p-median problem on a tree, In Proceedings of Oper. Res. Lett., (2007) 331-335. 